\title{
Editorial
}

\section{Diagnostic and prognostic markers in liver cirrhosis}

\author{
Salvador Augustin ${ }^{\mathrm{a}, *}$ and Joan Genescà ${ }^{\mathrm{a}, \mathrm{b}}$ \\ ${ }^{a}$ Liver Unit - Department of Internal Medicine, Hospital Universitari Vall d'Hebron, Institut de Recerca (VHIR), \\ Universitat Autònoma de Barcelona, Barcelona, Spain \\ ${ }^{\mathrm{b}}$ Centro de Investigación Biomédica en Red de Enfermedades Hepáticas y Digestivas (CIBERehd), Instituto de \\ Salud Carlos III, Madrid, Spain
}

Liver cirrhosis is the final stage of all chronic liver diseases. Although cirrhosis is a pathological concept, the diagnosis of this entity is frequently based on clinical criteria that mainly reflect the consequences of increased portal pressure. Once the diagnosis of liver cirrhosis is performed, the time to reach a clinical situation of end-stage liver disease and a need for liver transplantation might be quite long. In this process, patients progress from a compensated phase with no clinical complications to a decompensated phase, in which patients present the main clinical events in liver cirrhosis: variceal bleeding, ascites and renal failure, encephalopathy, and hepatocellular carcinoma.

This Special Issue of Disease Markers is dedicated to explore in eight chapters the importance of diagnostic tools and the role of different prognostic markers in the different stages and clinical complications of liver cirrhosis. The current knowledge is reviewed by eight selected invited groups of experts in the field.

In the first review, Albillos and García-Tsao (Classification of cirrhosis: The clinical use of HVPG measurements) examine the prognostic importance of portal hypertension in liver cirrhosis and propose several stages in cirrhosis based on the degree of portal hypertension and the presence of the clinical complications, indicating also practical aspects of the technique

${ }^{*}$ Corresponding author: Salvador Augustin, Liver Unit - Department of Internal Medicine, Hospital Universitari Vall d'Hebron, Institut de Recerca (VHIR), Universitat Autònoma de Barcelona, Barcelona, Spain. E-mail: saugustin@vhebron.net. to measure portal pressure. They provide a new and accurate risk-stratification classification with different stages that take into account clinical events, portal pressure information, outcome and the most important tools to assess prognosis at each stage.

Berzigotti and colleagues, in the second review (Non-invasive diagnostic and prognostic evaluation of liver cirrhosis and portal hypertension), concentrate on the non-invasive methods currently used in clinical practice for diagnosing liver cirrhosis and portal hypertension. They present easy-to-use and readily available techniques including laboratory parameters, transient elastography (measured by Fibroscan ${ }^{\circledR}$ ) and Doppler ultrasound. More complex, more expensive and less frequently used imaging methods consist of computed tomography scan and magnetic resonance imaging, while new technologies currently under evaluation and development are magnetic resonance elastography and acoustic radiation force imaging. They conclude that these non-invasive tools help to manage patients with cirrhosis and portal hypertension, provide valuable prognostic information, and avoid in some instances the use of more invasive techniques.

In their discussion of 'Prognostic markers in patients with ascites and hepatorenal syndrome', Nazal and Cárdenas provide an overview of the influence of the presence of ascites, hyponatremia and hepatorenal syndrome in the prognosis of liver cirrhosis. These complications are a direct consequence of the underlying circulatory dysfunction that occurs in patients with cirrhosis at an advanced stage, for which no validated 
prognostic markers are available. Prognostic factors related to liver dysfunction are very important and universally used (Child-Pugh score and the MELD score), both include variables that take into account liver and renal function parameters. The authors indicate that more studies are needed in order to define whether other variables of circulatory and renal dysfunction may improve the prognostic capability of these models.

In the next review, Villanueva and colleagues (Prognostic markers in patients with cirrhosis and portal hypertension who have not bled) consider all relevant diagnostic tools and prognostic markers in the setting of cirrhotic patients who have not yet presented a variceal bleeding. They differentiate two stages in this subgroup of patients: patients who do not have developed varices yet, the so-called pre-primary prophylaxis phase, and patients who already present varices, the primary prophylaxis phase. The different tools utilized to assess risk in these phases, especially the role of determining portal pressure, are being discussed by the authors. At the end, they also introduce the possible implications in future research in this area.

The prognosis and prognostic factors of acute variceal bleeding are discussed by Augustin and colleagues in the chapter entitled 'Prognostic evaluation of patients with acute variceal bleeding'. The aim of this review is to provide information that may help to understand why none of the available predictive models in this setting is routinely used in clinical practice, and to identify new ways to improve our current prognostic tools. To this purpose, the authors cover first some theoretical aspects that may serve as a framework to evaluate a prognostic model. Later in their article, specific issues about the current knowledge of the prognosis of the acute bleeding are revised.

Bañares and colleagues, in the sixth review (Prognostic markers in patients who have recovered from an acute variceal bleeding: role of HVPG measurement), critically assessed the role portal hemodynamic parameters in patients surviving a first episode of variceal bleeding, considering that they are at high risk of recurrent bleeding if remain untreated. The authors explain that risk of rebleeding is not homogeneous through the overall spectrum of bleeding patients, suggesting the need to stratify the rebleeding risk and to adjust the intensity of therapy according to this risk. In their article they review the utility of hepatic venous pressure gradient measurement in variceal rebleeding and its potential impact in tailoring therapy.

A unique complication of liver cirrhosis, hepatic encephalopathy, is discussed by García-Martínez and colleagues in their chapter about 'Prognostic assessment in patients with hepatic encephalopathy'. They explain that hepatic encephalopathy is a common complication of liver failure that is associated with poor prognosis, but the prognosis is not uniform and depends on the underlying liver disease. In patients with cirrhosis the prognosis of the episode of encephalopathy is usually dictated by the underlying precipitating factor. In acute-on-chronic liver failure, the most severe form of decompensation of cirrhosis, the prognosis depends on the number of associated organ failures. For the authors, the recurrence of encephalopathy is more common in patients who develop progressive deterioration of liver function and hyponatremia. They also indicate that bouts of hepatic encephalopathy may cause sequels that have been shown to persist after liver transplant.

The final article in the series, 'Diagnostic and prognostic molecular markers in hepatocellular carcinoma' by Mínguez and Lachenmayer, provide an extensive overview of the current situation and future perspectives for the integration of molecular data into clinical management of hepatocellular carcinoma. The authors explain how the molecular profiling of this tumour has led to a better understanding of its physiopathology and allowed developing novel therapeutic approaches (molecular targeted therapies). They also elegantly summarized all the information derived from reported genomic datasets, gene signatures derived from tumours and from the adjacent tissue, and epigenetic analyses (methylation and miRNA profiling). Finally, they indicate how this genetic and epigenetic information of the tumor and the surrounding tissue should be used to identify novel biomarkers and therapeutic targets in hepatocellular carcinoma, and to eventually design a more personalized medicine in this complication of liver cirrhosis. 


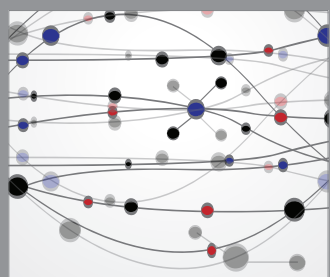

The Scientific World Journal
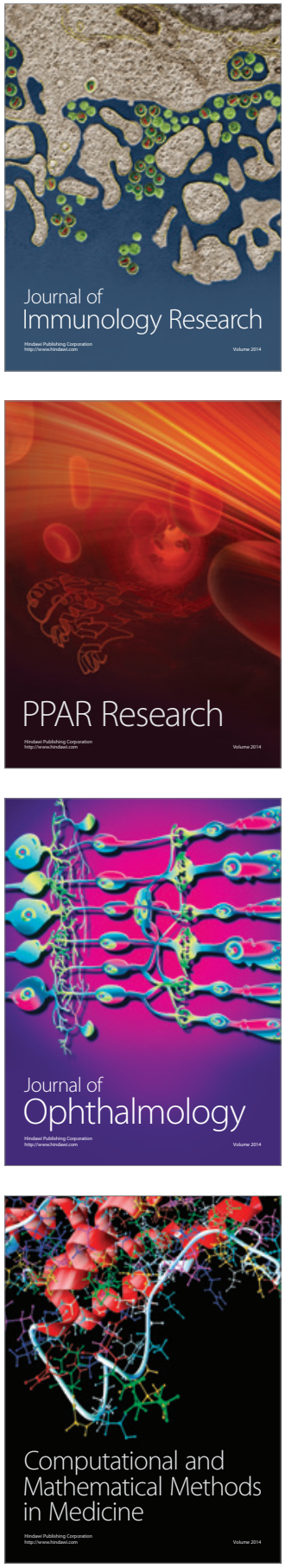

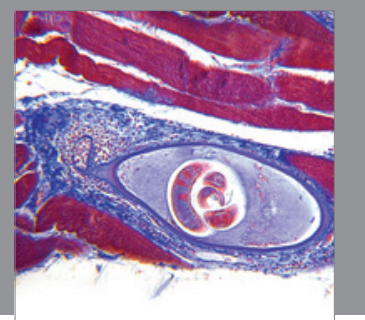

Gastroenterology

Research and Practice
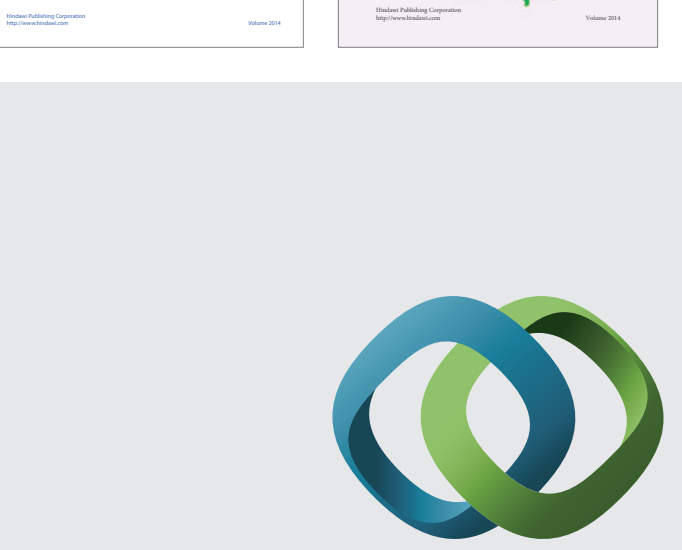

\section{Hindawi}

Submit your manuscripts at

http://www.hindawi.com
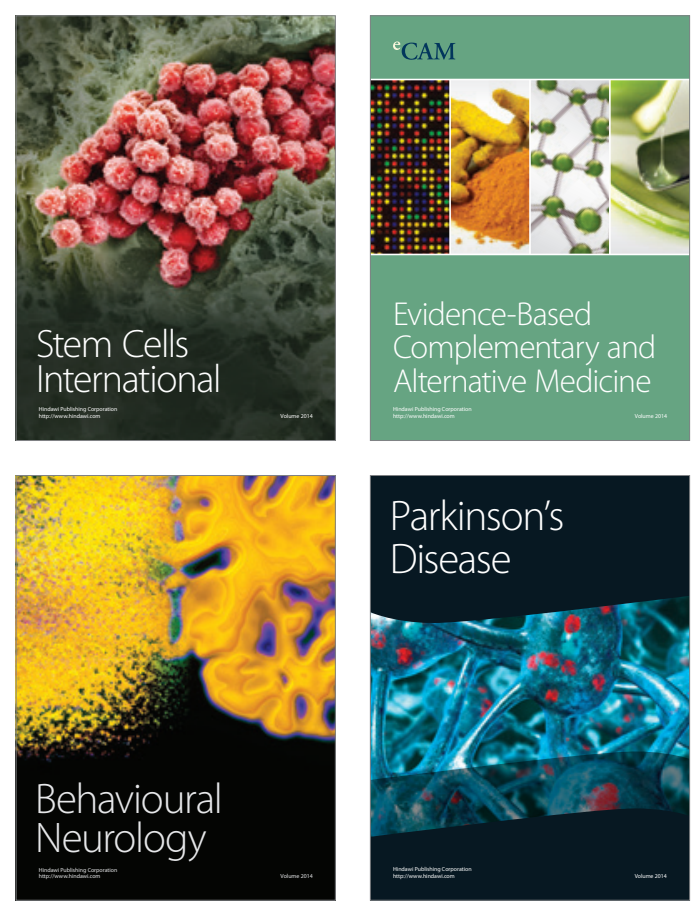

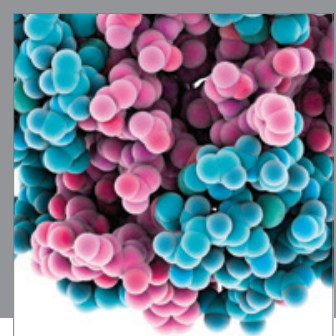

Journal of
Diabetes Research

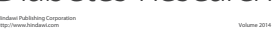

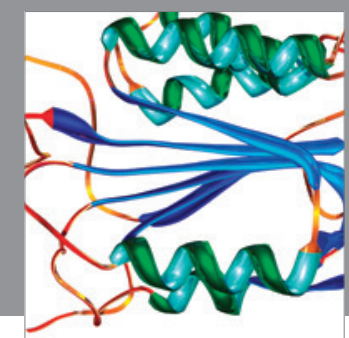

Disease Markers
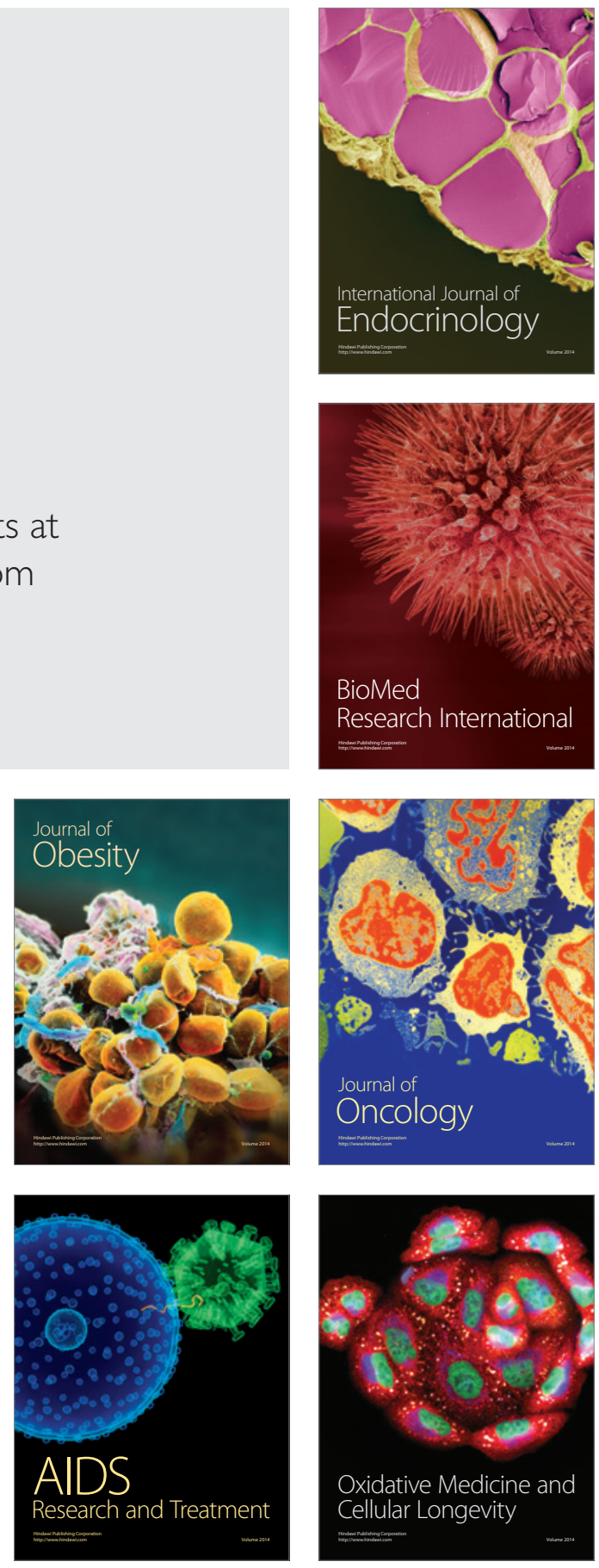